\title{
Investigación sobre Violencia de Género en la Pareja: Recomendaciones Prácticas
}

\author{
Roberta de Alencar-Rodrigues ${ }^{1}$ \\ Leonor M. Cantera \\ Departamento de Psicología Social de la Universidad Autónoma de Barcelona, \\ Barcelona, España \\ Carmen L. Ojeda Ocampo Moré \\ Departamento de Psicologia da Universidade Federal de Santa Catarina, Florianópolis, \\ Santa Catarina, Brasil
}

\begin{abstract}
Resumen
El objetivo de este trabajo es relatar la experiencia de búsqueda de participantes de una investigación cualitativa sobre violencia de género en la pareja contra mujeres inmigrantes latinoamericanas. Se discuten cuestiones como dificultades de la inserción en el campo, estrategias para lograr el muestro, aspectos éticos a tener en cuenta en estudios sobre violencia. Esas dificultades encontradas en el recurrido metodológico pueden ser un reflejo de los obstáculos que impiden que las mujeres busquen apoyo institucional. Ello lleva a entender que hay barreras comunes en el campo de investigación e intervención en violencia de género que merecen ser identificadas y superadas. Por último, se advierte sobre el impacto de las entrevistas sobre violencia en la vida de los(as) investigadores(as) y se sugiere que ellos(as) adopten estrategias para equilibrar su vida personal y profesional a través de actividades que puedan descargar la carga emocional producida a partir del trabajo con violencia.
\end{abstract}

Palabras clave: Investigación cualitativa, violencia de género, cuestiones éticas.

\section{Pesquisa sobre Violência de Gênero no Casal: Recomendações Práticas}

\section{Resumo}

O objetivo de este trabalho é descrever a experiência de busca das participantes de uma pesquisa sobre violência de gênero no casal contra mulheres imigrantes latino-americanas. As dificuldades de acesso ao campo de pesquisa, as estratégias para conseguir a amostra e os aspectos éticos em estudos sobre violência são discutidos. Essas dificuldades encontradas no percurso metodológico podem refletir os obstáculos que impedem que as mulheres busquem apoio dos serviços sociais. Isso mostra que existem barreiras comuns na área de investigação e intervenção em violência de gênero que devem ser identi-

Endereço para correspondência: Departamento de Psicología Social, Grupo de Investigación: Violencia en la Pareja y el Trabajo, Universidad Autónoma de Barcelona, Edificio B - Despacho B5/040, Bellaterra, Barcelona, España 08193. E-mail: roberta.alencar.rodrigues@gmail.com, leonor.cantera@uab.cat e carmen.more@ufsc.br Esta investigación fue financiada por el Programa AlBan, Programa de Becas de Alto Nivel de la Unión Europea para América Latina, beca n E07D401883BR - 2007/2010.

Las autoras agradecen a las instituciones, a los equipos de los servicios donde se ha realizado la investigación, a las participantes del estudio y a las personas profesionales Isabel Bianchi Sanchez, Olga Arisó Sinués, Patricia Jiron, Montserrat Paíno Lafuente, Imma Lloret, Lourdes Aramburu Otazu, Júlia Masip Serra, Anna Choy i Vilana por la ayuda prestada a la investigación. 
ficadas e eliminadas. Por último, chama-se atenção sobre o impacto das entrevistas sobre violência na vida dos(as) pesquisadores e sugere-se que eles(as) adotem estratégias para equilibrar a vida pessoal e profissional através de atividades em que possam descarregar a carga emocional gerada a partir do trabalho com violência.

Palavras-chave: Pesquisa qualitativa, violência de gênero, aspectos éticos.

\title{
Research about Gender Violence: Practical Guide
}

\begin{abstract}
This paper aims to describe the experience of finding participants for a qualitative research on gender violence against Latin American immigrant women. The challenges in reaching the research sample, strategies to achieve the aimed number of participants, as well as ethical aspects involved in studying gender violence are discussed in this work. The problem found on the methodology can reflect the obstacles that impede women to seek help. It seems that there are similar barrier in the field of research and intervention about violence that should be identified. Finally, advice is given on the impact produced by the interviews on the researchers' lives and we recommend that they adopt strategies in order to balance personal and professional life.
\end{abstract}

Keywords: Qualitative research, gender violence, ethical issues.

Diferentes estudios (Ellsberg \& Heise, 2007; Santos, 2009; Shirwadkar, 2004) coinciden en señalar sobre la dificultad para acceder a la muestra en trabajos sobre violencia de género en la pareja. Esas investigaciones con mujeres que han sufrido violencia de género en la pareja ponen el acento en la importancia del anonimato y la confidencialidad. A esto se añade la especificidad de la temática de violencia, la cual requiere que el equipo de investigación cuide de los efectos de la entrevista en la vida de las informantes y de los(as) investigadores(as). Teniendo en cuenta esas particularidades de las investigaciones sobre violencia, es preciso como en cualquier otro estudio, diseñar estrategias para acceder a las participantes adaptadas a distintas dificultades, con el fin de lograr un muestreo idóneo que permita responder a los objetivos de la investigación.

En este trabajo se pretende relatar la experiencia de búsqueda de participantes de una investigación sobre violencia de género en la pareja contra mujeres inmigrantes latinoamericanas y las estrategias utilizadas para obtener el muestreo. Para dar respuesta a los propósitos de este estudio, se ha elegido la metodología cualitativa, la cual se asienta en la perspectiva de que el conocimiento se alcanza a partir de los significados que las personas atribuyen a los eventos sociales (Creswell, 1998), los cuales son construidos a través de la relación dinámica entre el mundo real y el sujeto (Coyle, 2007).

En este sentido es importante mencionar algunas características del enfoque cualitativo para entender las consecuencias que esta metodología favorece. La característica de objetividad en el enfoque cualitativo, según Martins (2004), tiene que ver con los criterios establecidos por quien investiga por lo que respecta al problema de investigación. Por ello, el tamaño de la muestra en el contexto cualitativo, según esta autora, no tiene que ver con la noción estadística de muestra de la metodología cuantitativa.

A esto hay que añadir que Martins (2004, p. 292) afirma que la "neutralidad no existe", pues hay que reconocer la influencia del bagaje de experiencias y conocimiento del y de la investigador(a). Así, se identifica que la noción de neutralidad del abordaje cualitativo favorece la investigación sobre violencia, pues, según Penso, Almeida, Brasil, Barros, y Brandão (2010), no hay neutralidad en la relación entre la persona profesional y la víctima de violencia cuando se interviene con violencia, pues ambas son mujeres y están afectadas por las cuestiones de género. Siguiendo esta lógica, se puede 
decir, que la neutralidad tampoco ocurre en la relación entre investigadora y entrevistada en investigaciones sobre violencia.

Resulta importante relatar esta experiencia para dar a conocer los elementos que debería cumplir toda investigación científica sobre violencia de género $y$, se espera que esta aportación pueda ser de utilidad para la formación de investigadores(as) en este ámbito.

Teniendo en cuenta las recomendaciones sobre planteamiento de investigación cualitativa (Alves, 1991), inicialmente se realizó la revisión de literatura con el objetivo de identificar lagunas que guiaron la formulación de la pregunta de investigación y de los objetivos. La pregunta inicial y los objetivos sufrieron ajustes conforme se iban analizando los datos y emergían nuevos temas que se debían explorar. Además, se buscó hablar con algunas personas profesionales de los servicios para examinar qué hace falta saber sobre el tema de violencia de género en la pareja que padece el colectivo inmigrante. Con ello, se pudo verificar lo que ya se sabía sobre el maltrato que acomete a las mujeres inmigrantes, pues según Ellsberg y Heise (2007), se debe prestar atención para no investigar temas que no aportan conocimiento para evitar situaciones de victimización secundaria que llevan a las mujeres a repetir su historia innúmeras veces.

Todo ello fue posible gracias al diseño flexible de la investigación cualitativa, que permite que se cambie la dirección y se redirija la recogida de datos con el objetivo de efectuar una nueva inserción en el campo (Miles \& Huberman, 1984). Así se pudo formular el propósito del estudio que fue dar respuesta a la pregunta " ¿Qué relación guarda el hecho de ser inmigrante y la violencia de género en la pareja?" pues la literatura evidencia la escasez de trabajos empíricos sobre la situación de mujeres inmigrantes latinoamericanas que padecen maltrato en el Estado Español.

Teniendo en cuenta que Schraiber, Lucas d'Oliveira, y Couto (2009) afirman que la objetividad en el estudio cualitativo exige que la persona investigadora aclare las decisiones realizadas durante el proceso investigativo para asegurar la confiabilidad de la producción del conocimiento, se pasa a analizar a continuación la trayectoria realizada para acceder a la población diana, describiendo las estrategias para lograr la muestra y presentar los pasos seguidos para beneficio de futuros estudios. Posteriormente, se define el muestreo adoptado, se caracterizan las participantes, se explica el instrumento utilizado y se detalla el proceso de recolección de datos.

\section{Inserción en el Campo}

El estudio fue realizado en la ciudad de Barcelona, cuya población se estima en 1.621.537 habitantes (Ayuntamiento de Barcelona, 2011). La inserción se realizó a partir de dos instituciones públicas. En una de ellas, se presentó el proyecto y se solicitó ayuda formal para la investigación. A partir de esta visita, se obtuvieron informaciones sobre las entidades especializadas en Barcelona en la atención de mujeres inmigrantes que sufren violencia de género en la pareja.

En la segunda institución, se realizó una visita a las personas profesionales de este servicio quienes informaron a la investigadora sobre el Circuito Barcelona contra la Violencia y le pusieron en contacto con personas profesionales de los servicios sociales de Barcelona, escribiendo a sus contactos, presentando la procedencia y formación de la investigadora y explicando los objetivos de su proyecto. La disposición del segundo Centro para contribuir a este estudio fue fundamental para facilitar el inicio de la recogida de datos de esta investigación. La facilitación de contactos profesionales y de la guía de entidades de Barcelona que trabajan directamente con la violencia de género en la pareja, brindaron sin duda alguna la apertura de un abanico de posibilidades de mayor acceso a los servicios de atención a las mujeres que sufren violencia de género en la pareja.

Una vez obtenido el conocimiento de esos servicios, se contactó a través del teléfono y del correo electrónico con 64 instituciones para presentar la investigación. De estas, 15 facilitaron la oportunidad de llevar a cabo una reunión en la cual la investigadora pudo presentar el proyecto y la guía de entrevista de la investigación, acla- 
rar dudas y reforzar la solicitud formal de colaboración con el presente estudio. También se utilizó el boca a boca, es decir, la investigadora habló en muchos escenarios sobre esta investigación, especialmente en eventos sobre la temática del estudio, y pidió que si conocían a alguien que quisiera participar en el estudio, por favor, le pusieran en contacto con ella. Para ello, facilitaba sus señas personales mediante folleto de invitación.

En el proceso de acceso a las participantes, cabe destacar las respuestas de algunas instituciones ante la invitación para participar en este estudio, las cuales arrojan luz sobre la dificultad de lograr una muestra numerosa y sobre la complejidad de la temática de la violencia de género en la pareja.

A lo largo de todo el contacto con las instituciones, se trató de pedir que eses servicios difundiesen el estudio y facilitasen el número de teléfono y el correo electrónico de la investigadora a las usuarias inmigrantes. A las personas profesionales se les solicitó que preguntasen a las mujeres interesadas en contribuir con el estudio si ellas preferían llamar a la investigadora o autorizaban a la institución a conceder su número de teléfono a la investigadora. Partiendo de la base de que mundialmente el tema de la violencia de género es delicado y requiere protección y seguridad para las víctimas, se reconoció que no sería ético y respetuoso pedir el contacto de las usuarias inmigrantes.

Aunque la investigadora haya adoptado la postura de solicitar dentro de lo posible que las personas profesionales explicasen la investigación y facilitasen el contacto de la investigadora a las usuarias interesadas, recibió en repetidas ocasiones respuestas negativas debido al sistema de seguridad y protección de datos. Ante tal situación, la investigadora insistió en que su objetivo era que las mujeres inmigrantes que han padecido violencia tuviesen conocimiento de la investigación y desde su propio criterio decidiesen participar o no. Siempre siendo ellas quienes decidieran a partir de lo que las profesionales les contaran. Sin embargo, algunas responsables de las instituciones explicaban a la investigadora que ya llevaban muchas actividades y presentar su propuesta a las usuarias les implicaría más trabajo. Ante a esta explicación, se entendió la postura de las instituciones dado que se solicitaba una petición más allá de las tareas que las personas profesionales ya cumplían.

Esas dificultades para acceder a mujeres que sufren o han sufrido maltrato apoyan lo encontrado en la revisión de la literatura sobre el tema (Santos, 2009; Shirwadkar, 2004). La primera autora llamó a 40 mujeres que habían interpuesto denuncia en una comisaría especializada en la mujer en Brasil, pero sólo 10 aceptaron participar en su estudio sobre la caracterización de la violencia sufrida por mujeres y del soporte ofrecido por la red de servicios de una ciudad brasileña. La segunda investigadora logró entrevistar a ocho mujeres inmigrantes indias que habían vivido violencia de género en la pareja en Canadá debido a los desafíos para acceder a esta población diana.

Además del sistema de seguridad y protección de datos, otra razón que puede explicar los obstáculos al realizar estudio sobre violencia son, según Ellsberg y Heise (2007), las actitudes reacias de las personas profesionales de las instituciones ante los(as) investigadores(as). De acuerdo con esas autoras, esa resistencia puede ser producto de experiencias negativas con investigadores(as) que buscan los servicios sólo para acceder a la muestra. A continuación, se registra la perspectiva de Ellsberg y Heise (2007, p. 64):

Muchos organismos no gubernamentales habían tenido experiencias que les hacían sentir desconfianza ante solicitudes de "colaborar" en proyectos de investigación. Señalaron que investigadores parecen más preocupados con su proprio desarrollo profesional que con el proprio bienestar de las personas a quienes entrevistan.

Se comparte la idea de las autoras de que la resistencia de instituciones se debe a la falta de ética de algunos(as) entrevistadores(as) que están más preocupados con sus intereses profesionales que por quiénes atienden. Sin embargo, se considera que los servicios deberían incorporar políticas que faciliten las investigaciones, pues según el II Plano Nacional de Políticas para as 
Mulheres (Secretaria Especial de Políticas para as Mulheres, 2008) planteado por el gobierno brasileño, resulta importante que esos organismos incorporen las contribuciones de los estudios sobre violencia.

Otro elemento que se debe considerar en el análisis de las dificultades para obtener un muestreo idóneo es la situación de vida de las mujeres inmigrantes. Las condiciones del proceso migratorio (falta de redes de apoyo, tipo de empleo) les hacen más difícil la tarea de compaginar la vida laboral y familiar, por lo que resulta más probable que las posibilidades de lograr tiempo para conceder una entrevista se reduzcan. De igual manera, algunas participantes del estudio revelaron que ahora percibían que podían hablar sobre la situación de violencia de género en la pareja, lo cual anteriormente les costaba mucho. Este dato lleva a entender que en la investigación sobre violencia de género se debe "tener en cuenta la particularidad de cada mujer, la fase del proceso que está atravesando" tal y como recomienda Romero (2010, p. 191) al hablar sobre la intervención en violencia. Esta atención hacia el estado emocional y el momento de vida de la mujer revela que el objeto violencia, como afirman Schraiber et al. (2009, p. 207), "tiene especificidades para objetivación asociadas a las emociones que la violencia despierta, las cuales exigen cuidados metodológicos de naturaleza ética".

Teniendo en cuenta que Shetty y Kaguyutan (2002) explican que experiencias previas en el país de origen y las características institucionales (ubicación, habilidades lingüísticas y etnicidad del equipo) son factores con las que las mujeres inmigrantes se encuentran para buscar apoyo institucional, se puede esperar que el número reducido de mujeres que reciben tratamiento en los servicios refleje esas barreras. A esto Konrad (2003) agrega la falta de apoyo con el transporte y el horario de las reuniones en servicios que no coincide con la disponibilidad de las mujeres. $\mathrm{La}$ consideración de estos aspectos sugiere que las dificultades encontradas en el recurrido metodológico de investigaciones sobre violencia pueden ser un reflejo de los obstáculos que impiden que las mujeres busquen tratamiento en entidades especializadas en la atención de mujeres que sufren violencia de género en la pareja.

Debido a estos motivos, se desprende que las usuarias de los servicios a las que se les informó sobre la investigación pueden haber decidido no participar en el estudio por cuestiones de tiempo o por no encontrarse en el momento adecuado para compartir su historia. Con respecto a este aspecto, se pidió que las personas profesionales explicasen la investigación a aquellas mujeres que considerasen que se encontraban en un momento del proceso en el cual hablar sobre la situación de violencia de género no resultase tan doloroso a fin de evitar la revictimización. Además, se planteó que el beneficio de participar de la investigación sería que el testimonio de las participantes podría servir de modelo a otras mujeres que padecen violencia; para que pudiesen conocer que hay estrategias para salir de la situación de violencia. Con ello, se buscó asegurar que la experiencia de conceder entrevista sobre la situación personal de maltrato tuviese sentido para las participantes del estudio.

Otra consideración importante es que entre el conjunto de contactos facilitados por las personas profesionales, un total de siete mujeres acabaron no participando en la investigación por los motivos que se explican a continuación:

1. Dos mujeres avisaron antes de la sesión de entrevista de que no se encontraban en un momento adecuado y que se pondrían en contacto con la investigadora más adelante. Así, se les ofreció la posibilidad de realizar la entrevista en otro momento pero, aún así, ellas dijeron que preferían llamar a la investigadora. Ante esta respuesta y respetando el derecho de las participantes, se optó por no insistir y no llamarlas.

2. Dos mujeres no acudieron a la sesión de entrevista, aunque habían aceptado participar en el estudio mediante contacto telefónico. Se les llamó para preguntar si les había pasado algo, pero no contestaron. Ante esta situación, se respetó el desistimiento de la mujer en no participar en el estudio.

3. Tres no contestaron a la llamada de teléfono. Todo ello configuró las especificidades del trabajo de campo en el ámbito de la violencia de 
género en la pareja, que implico un acceso limitado a las participantes. Este dato apoya lo encontrado por la investigadora antropóloga Mercedes Fernández-Martorell en su estudio sobre hombres agresores y que reflejó posteriormente en la película titulada ¿No Queriais Saber por qué las Matan? POR NADA (2009), producto de la investigación llevada a cabo en la Universidad de Barcelona bajo su coordinación. Esta autora explica cómo tardó 3 años, asistiendo a más de 700 juicios, para lograr sólo cerca de 20 entrevistas con hombres autores de violencia. Lo que parece evidente es que el estigma social de algunos temas de investigación puede generar problemas para encontrar participantes (Warren, 2001).

En este marco de adversidades, en el presente estudio la investigadora buscó estrategias para solucionar los obstáculos que estaban en su poder controlar realizando acciones como: facilitar transporte y comida a las mujeres entrevistadas dependiendo del caso, buscar una persona adulta para la atención y cuidado de sus hijos e hijas (lo que se conoce en el contexto de la investigación como canguro) mientras se realizaba la entrevista y solicitar el apoyo formal de los centros cívicos y asociaciones de inmigrantes pidiéndoles sala para realizar las entrevistas.

A pesar de todas las dificultades descritas anteriormente hay que reconocer, desde luego, que el hecho de que la investigadora sea mujer pudo haber facilitado el acceso a la población diana, pues según Reinharz y Chase (2001, p. 232), "el género de la entrevistadora afecta la voluntariedad para compartir experiencias personales". A esto se une el origen latinoamericano de la misma, que puede haber ayudado al acceso a las participantes, pues esta autora revela que la accesibilidad a la muestra y la comprensión de las participantes es más fácil cuando la investigadora y las participantes comparten ubicaciones sociales similares. Tal reflexión ha sido ya realizada por Shirwadkar (2004), quien explica que fue aceptada en la comunidad inmigrante india en Canadá por ser también proveniente de India.

La experiencia de inserción en el campo revela que la violencia de género sumada a la población escogida (mujeres inmigrantes) constituye un tema delicado, ya que se cruza, según Schraiber et al. (2009), dos condiciones que estigmatizan: padecer violencia y pertenecer a determinadas etnias. Aunque esas autoras recomienden que se evite combinar violencia con otros temas, se considera que hay particularidades de la violencia vivida por las mujeres inmigrantes que necesitan ser visibilizadas para una mejor atención y respuesta a sus necesidades y realidad.

Conviene recordar que la dificultad de acceder a las participantes y las restricciones contextuales que se impusieron en el proceso de inserción en el campo conllevaron la necesidad de ajustar la manera de recoger los datos (Creswell, 1998). Esto influenció en el tipo de muestreo adoptado en la investigación, que se explica en la próxima sección.

\section{Muestreo}

La muestra fue compuesta por 14 participantes que cumplieron tres criterios de elegibilidad: ser mujer inmigrante latinoamericana, haber sufrido violencia de género en la pareja en España y haber recibido ayuda por parte de algún servicio de la red de recursos ofrecidos por el Ayuntamiento de Barcelona. Se caracterizó por ser un muestreo intencionado, dado que las personas participantes fueron seleccionadas de acuerdo con criterios predeterminados por el objetivo de la investigación (Guest, Bunce, \& Johnson, 2006).

Las 14 participantes migraron desde Bolivia (1), Brasil (3), Chile (1), Colombia (1), Ecuador (3), El Salvador (1), Paraguay (1), Perú (2) y Republica Dominicana (1). Han vivido en España de 8 meses hasta 20 años y tenían entre 23 y 45 años. Casi todas (12/14) tenían hijos(as). Seis estaban solteras en el momento del estudio, cuatro estaban divorciadas, tres vivían con su pareja y una estaba casada.

El número de participantes fue determinado por el conjunto de dificultades descritas anteriormente y su valor se ampara al tener en cuenta la literatura internacional sobre el tamaño de la muestra en contexto el cualitativo, que se define según la saturación teórica. Dicho concepto, se- 
gún Bauer y Aarts (2004, p. 39), es el "criterio de finalización" de la muestra que se alcanza cuando, conforme a Gaskell y Bauer (2004, p. 485) nuevos datos añadidos al análisis "no brindan nuevas observaciones".

Francis et al. (2009) y Guest et al. (2006) ponen en tela de juicio el concepto de saturación teórica. Las primeras autoras revisaron 24 libros de metodología de investigación y siete bases de datos e identificaron que no hay una guía práctica que calcule el tamaño de la muestra y explique cómo determinar la saturación, por lo que concluyen que el concepto de saturación tiene un "significado difuso y vago" (Guest et al., 2006, p. 65). De igual manera, los últimos autores revisaron artículos de los periódicos Social Science y Medicine durante 16 meses. Entre los 18 documentos encontrados que mencionaron saturación de datos, Francis et al. (2009) constataron falta de claridad por lo que respecta a la decisión del criterio de finalización de la muestra.

Tomando en consideración esta laguna encontrada en la revisión teórica, Guest et al. (2006) se propusieron investigar "cuántas entrevistas son necesarias para alcanzar el agotamiento temático y la variabilidad dentro del conjunto de los datos". Partiendo de esta pregunta de investigación, Guest et al. entrevistaron a $60 \mathrm{mu}-$ jeres que cumplían por lo menos tres criterios: (a) tener 18 años de edad o más; (b) haber tenido sexo vaginal con más de un compañero en los últimos tres meses, y (c) haber tenido sexo vaginal tres o más veces en la media semanal. Una vez analizadas las 60 entrevistas, Guest et al. concluyeron que la saturación se obtuvo después de haber analizado el conjunto de las primeras 12 entrevistas. Después del examen cualitativo de las primeras 12 entrevistas, las autoras pudieron crear el 92\% (100) del total (109) del número de los códigos. Los restantes nueve nuevos temas emergieron de manera infrecuente después del conjunto de 12 entrevistas.

De igual manera, Francis et al. (2009) establecen principios para determinar la saturación teórica. En primer lugar, esos teóricos recomiendan que se establezca una muestra inicial de análisis, en la cual el tamaño depende de la complejidad de las cuestiones de investigación, de la guía de entrevista, de la diversidad de quienes participan y del tipo de tratamiento de los datos. En segundo lugar, estos autores sugieren que una vez realizada la muestra inicial, se determine el número de entrevistas que se realizarán sin la aparición de temas emergentes, por lo que denominan este proceso de criterio de finalización. Para ello, ejemplifican una muestra inicial de 10 participantes en la cual la saturación teórica se logra mediante la inclusión de más entrevistas, donde las últimas tres consecutivas no deben tener contenido nuevo. En tercer lugar, proponen que el análisis sea conducido por dos codificadores independientes. Por último, aconsejan que se aclare el método de saturación para que las personas lectoras puedan evaluar la prueba. Sin embargo, reconocen que no siempre es posible aplicar todos esos principios debido a las restricciones contextuales de un proceso de investigación.

Asimismo, Ellsberg y Heise (2007), expertas en investigación sobre violencia, afirman que no hay reglas estrictas cuanto al tamaño de la muestra en la investigación cualitativa, pues ello depende de factores como el objetivo del estudio, lo que se puede hacer con el tiempo y los recursos que se tiene.

Teniendo en cuenta las orientaciones de Ellsberg y Heise (2007), Francis et al. (2009) y Guest et al. (2006), se considera que la muestra de 14 participantes fue adecuada para satisfacer los propósitos de la investigación, que consistió en entender las percepciones y experiencias comunes de las mujeres inmigrantes latinoamericanas usuarias de servicios sociales en la ciudad de Barcelona respecto a la vivencia de violencia de género en la pareja. Además, se considera que lo más importante es "el potencial de caso para ayudar al investigador en el desarrollo de comprensiones teóricas sobre el área estudiada y no el número de casos" como bien defienden Taylor y Bogdan (1994, p. 108).

Cabe mencionar que a partir de la séptima entrevista, se observó la recurrencia temática en relación con los objetivos del presente estudio, y el número final del muestreo logró saturación teórica por lo que respecta a los objetivos específicos. 
Se utilizó la entrevista semi-estructurada, pues permite a la entrevistadora realizar preguntas abiertas y preguntas específicas. Es decir, las preguntas abiertas son importantes dado el carácter exploratorio de este estudio (Miles \& $\mathrm{Hu}-$ berman, 1984), en el cual la investigadora busca entender "sobre lo que es importante en la mente de las participantes" (Taylor \& Bogdan, 1994, p. 114), y siempre que las preguntas específicas garanticen que se exploran diferentes temáticas con cada participante. Para ello, Charmaz (2001, p. 679) sugiere el uso de preguntas abiertas que sean suficientemente "generales" como para contemplar una gama de experiencias y preguntas cerradas que sean "estrechas" para obtener experiencias específicas de las participantes.

\section{Proceso de Recogida de Datos}

La recopilación de los datos siguió dos etapas. La primera se entrevistó a siete mujeres. Con ello, se revisó la pregunta previa de investigación y se desarrollaron otras cuestiones teniendo en cuenta las preocupaciones planteadas por esas primeras entrevistadas. En la segunda etapa, se buscó a otras participantes que pudiesen dar respuesta a esas cuestiones y refinar la teoría previa, y para ello, se entrevistó a otras siete mujeres. A continuación, se detalla cada etapa del proceso de recogida de datos.

Inicialmente se recurrió al personal responsable de 64 instituciones. De las cuales, se obtuvieron 15 respuestas favorables para acordar una reunión para presentar el proyecto y la guía de entrevista de la investigación, aclarar dudas y reforzar la solicitud formal de colaboración con el presente estudio. En general, las personas profesionales de las instituciones se comprometieron a explicar la investigación a las usuarias inmigrantes y aquellas que manifestaban interés en contribuir con el estudio autorizaban a la institución a facilitar su contacto a la investigadora. Sin embargo, hubo casos en que las propias usuarias inmigrantes se pusieron en contacto con la investigadora por correo electrónico o teléfono.

Posteriormente se contactó vía teléfono o correo electrónico con las posibles participantes, se hizo una presentación personal de la investi- gadora y se reiteró el propósito del estudio para identificar y aclarar dudas, se explicó el proceso de la sesión (entrevista), se les informó de la duración de la entrevista (1-2 horas), se abordaron las cuestiones de confidencialidad y anonimato y se consultó sobre el interés de participación. Tras la aceptación, se acordó una fecha y un local según la disponibilidad de la participante y se les ofreció ayuda para transporte, comida y cuidado de sus hijos y/o hijas, dependiendo de su situación particular. Con respecto a este último, resultó importante contar con el apoyo de integrantes del equipo de investigación para el cuidado de los(as) niños(as) de las participantes mientras ellas concedían entrevista en una sala solo con la investigadora.

Antes de cada sesión, se reservó el espacio para realizarla. Se buscaron lugares donde se garantizara la seguridad, la privacidad y tranquilidad para poder llevar a cabo la entrevista sobre un tema doloroso, complejo y delicado a la vez. Se procuró que el espacio para llevar a cabo la entrevista, además de guardar lo antes dicho, fuera acogedor y tuviera, para beneficio de la mujer entrevistada, pañuelos desechables y agua para beber. Para ello, se solicitó ayuda en los servicios sociales con los que las participantes ya estaban familiarizadas y se sentían cómodas. En el caso de que esos servicios no pudiesen conceder el espacio para la realización de las entrevistas, se solicitó apoyo de: una asociación de inmigrantes, de los centros cívicos y de los puntos de información a las mujeres (PIAD); buscando y vigilando, siempre, que esos lugares fueran de fácil acceso para las participantes.

Por lo que respecta a la conducción de las sesiones, inicialmente, se les agradeció la aceptación de la invitación a participar del estudio, se repitieron los propósitos de la investigación, se reforzó el compromiso de anonimato y confidencialidad, se aclararon dudas, se les pidió consentimiento del uso de información para fines científicos y académicos y se les solicitó permiso para usar la grabadora con el fin de registrar la entrevista. Acto seguido, se utilizó la guía de entrevista y al final se les agradeció su contribución y tiempo. Por lo que respecta al agradecimiento de participación en el estudio, se buscó subrayar 
la capacidad de resistencia de la mujer a la situación de opresión que le permitió poner fin a la situación de violencia. Conviene mencionar que las participantes al final de sesión también agradecieron "la oportunidad de desahogo", lo que sugiere, según Ellsberg y Heise (2007), que el momento de entrevista puede configurar una experiencia transformadora al revelar la historia de trauma. Según esas autoras, el hecho de abordar la violencia transmite el mensaje a las participantes de que no es un tema vergonzoso y merece ser estudiado.

En general, la duración de las entrevistas fue de 1 hora a 2 horas, pues se buscó tener en cuenta que menos de una hora no era suficiente para explorar muchos temas y más de dos horas les dejaría exhaustas (Taylor \& Bogdan, 1994). En el caso de las participantes que tenían premura de tiempo al cabo de una hora, pero aún manifestaban buena voluntad para hablar sobre sus experiencias y expresar sus sentimientos, se respetó su límite y se les ofreció la posibilidad de concluir la entrevista en otro momento. Eso posibilitó realizar los ajustes necesarios, resignificar temas y aclarar cuestiones en un segundo momento de encuentro.

En la primera etapa de recogida de datos, se analizaron los datos simultáneamente, $\mathrm{y}$, con ello, se identificaron lagunas en las entrevistas que necesitaban ser contestadas mediante nueva inserción en el campo (Charmaz, 2001). Así, se decidió ampliar la búsqueda de participantes a pesar de las dificultades encontradas previamente. Para ello, se buscó establecer comunicación con profesionales a través de la participación en los seminarios sobre la temática de violencia de género en la pareja e inmigración, con lo cual se obtuvieron más contactos y se pudieron realizar siete entrevistas más.

Con este recorrido, se esbozaron las características principales de las dos etapas de recogida de datos. A continuación, se describen algunas particularidades que se deben tener en cuenta en el proceso de recogida de los datos.

Particularidades en el Trabajo de Campo. Se debe tomar atención especial a la seguridad de las mujeres entrevistadas y de la persona que realiza la entrevista. Para ello, resulta importan- te evitar realizar la entrevista en la casa de las participantes y de preferencia recoger los datos en algún servicio o asociación que ellas estén familiarizadas o ya frecuenten. Durante la inserción en el campo, hubo una situación en que una candidata a participante se puso en contacto con la investigadora para cancelar la cita, pues su pareja se enteró de la invitación del estudio y dijo que le perseguiría para estar presente en la entrevista. Teniendo en cuenta esta situación, se debe tener el cuidado de escoger un lugar seguro para la recogida de datos como una institución en la cual se pueda pedir ayuda del personal de la seguridad en caso de algún incidente.

En general, se pudo observar que hubo participantes que fueron menos comunicativas $y$ que no iban más allá de las preguntas, por ello, fue importante empezar la entrevista con preguntas más abiertas y luego identificar el momento oportuno para realizar preguntas específicas. Para ello, se buscó realizar apuntes para indagar sobre puntos que no habían emergido previamente.

Otro aspecto del proceso de recopilación de datos fue ofrecer una escucha incondicional, incluso cuando las participantes hablaron de temas que no estaban contemplados en la guía de entrevista, pues resultó necesario no interrumpirlas aunque aparentemente lo que estaban relatando no guardaba relación con el tema tratado. En este punto, conviene mencionar que Romero (2010, p. 193) advierte sobre el problema en entender las mujeres que padecen violencia, pues "ellas pueden tener dificultades en expresar lo que les pasa, o pueden hacerlo de forma disociada, caótica o incongruente". No obstante, dado el carácter focalizado de las entrevistas y las limitaciones de tiempo, se buscaba que las participantes volviesen al tema de la entrevista diciéndole, por ejemplo: "Me gustaría volver a algo que usted dijo anteriormente", según orientan Taylor y Bogdan (1994).

Se ha de señalar también que el uso de expresiones en castellano características del país de origen de las entrevistadas dificultó algunas veces la comprensión del relato por parte de la investigadora. Por ello, se pidió una clarificación repitiendo la expresión que no se entendió y ha- 
ciendo la pregunta: “¿podría decir qué significa esa expresión para usted?”. De igual manera, se solicitaron ejemplos de las informaciones que no se entendían tal y como sugieren Taylor y Bogdan (1994).

La investigadora tuvo cuidado con los aspectos relacionados con la violencia de género en la pareja. Así, adoptó una postura que consistía en identificarse inmediatamente cuando las mujeres contestaron el teléfono, pues se consideró que las llamadas telefónicas de personas desconocidas podrían provocarles tensión si las participantes suponían que el interlocutor fuera la ex pareja agresora.

Cabe señalar de manera reiterativa que trabajar e investigar sobre la violencia de género en la pareja es un tema complejo, mucho más si se añade la variable inmigración. Mostrar la violencia de género en su dinámica de marginalidad del contexto migratorio incrementa sustancialmente la complejidad teórica y metodológica.

\section{Aspectos Éticos de la Investigación}

La investigación siguió los planteamientos éticos establecidos por la American Psychological Association (APA, 2010). Las mujeres inmigrantes latinoamericanas concedieron entrevistas voluntariamente, con consentimiento informado. Tanto en el contacto telefónico como en el primer momento de la entrevista, se les explicaron de manera general los propósitos del estudio, el tratamiento de anonimato y confidencialidad de los datos y el carácter voluntario de la participación en el estudio. Además, se les aseguró el derecho de desistir de participar en cualquier momento de la investigación.

Todo ello configura no sólo un conjunto de medidas de cuidado en relación con la persona participante en la investigación, sino también una manera de establecer una relación de comunicación próxima entre la investigadora y la participante (Creswell, 1998; Reinharz \& Chase, 2001; Taylor \& Bogdan, 1994). Con ello, la investigadora expresó su interés en las experiencias de las participantes, delimitando fronteras en la relación de investigación (Reinharz \& Chase, 2001). Es decir, la propuesta de la investiga- dora fue escuchar las experiencias de vida de las participantes.

Cabe señalar que en el proceso de entrevista existieron algunas situaciones en las cuales la investigadora tuvo que ofrecer apoyo, dado el carácter movilizador de la temática de la violencia de género en la pareja. En este caso, se procuró informarles sobre la red de recursos disponibles en Barcelona a las mujeres que padecen o han sufrido violencia de género en la pareja y ofrecerles referencias de profesionales especializados en la temática, pues como afirman Ellsberg y Heise (2007) es una obligación ética de la persona investigadora facilitar informaciones útiles y un listado de recursos en la comunidad. De igual manera, la investigadora les facilitó una tarjeta con teléfono móvil en el caso de que necesitasen alguna información y para evitar revictimización.

En este contexto de entrevista, Schraiber et al. (2009) llaman la atención sobre el sentimiento de impotencia expresado por los(as) investigadores(as) debido a la inseguridad que sienten al no saber si la acogida a las víctimas de violencia tendrá seguimiento o no. Por ello, cabe destacar que el criterio de seleccionar las participantes en la red de servicios fue una medida de protección, considerando que la temática de violencia de género en la pareja puede suscitar ansiedad, la cual debe ser apoyada.

Ante a los estados de angustia de las participantes, Ellsberg y Heise (2007) advierten que el o la investigador(a) cumplan su función de entrevistador(a) y tomen cuidado para no pasar a actuar como orientador(a). Este dato apoya la recomendación de Schraiber et al. (2009) quienes afirman que se debe acoger la persona que revela la experiencia de violencia y mantener la postura de investigación con el objetivo de evitar que la situación de entrevista se transforme en terapia.

Según Ellsberg y Heise (2007), una obligación ética que tienen los(as) investigadores(as) es aportar las conclusiones de sus estudios para el desarrollo de políticas públicas e intervenciones. En el caso de esta investigación, la investigadora se puso a disposición de las instituciones para devolución de los hallazgos.

Ellsberg y Heise (2007) advierten sobre la carga emocional en la que los(as) investiga- 
dores(as) están expuestas al escuchar historias de dolor físico y de degradación de las mujeres. Todo ello, según esas autoras, puede conllevar a consecuencias emocionales y personales para la vida de los(as) entrevistadores(as) que deben estar atentos para que las emociones provocadas durante las entrevistas no respinguen en sus relaciones personales.

El hecho de ser testigo del testimonio de la víctima tanto en investigación sobre violencia de género cuando en intervención sobre esta temática lleva a entender que el impacto que la escucha sobre la violencia genera en la subjetividad del terapeuta y del investigador es un tema que cobra importancia y no puede ser ignorado.

Por ello, Herman (2004) recomienda que las personas profesionales que trabajan con la temática de violencia violencia tengan equilibrio entre su vida personal y profesional. Ellsberg y Heise (2007) advierten que los equipos de investigación faciliten oportunidades de desahogo para que sus integrantes puedan expresar sus sentimientos y dudas, pues, según ellas, eso no solo contribuye a soportar la carga emocional del proceso de realización de entrevistas, sino aporta cualidad a la obtención de datos. Para estas autoras, resulta importante que se discuta sobre la violencia, el impacto del contenido de las entrevistas en la vida de los(as) investigadores(as) y se planteen las experiencias de maltrato de los(as) miembros(as) del equipo de investigación durante la capacitación de los(as) entrevistadores(as). Para ello, ellas recomiendan que se organicen encuentros separados para facilitar la expresión de emociones y para supervisar cuestiones técnicas del estudio. El estudio conducido por Penso et al. (2010) revela que estrategias como: realizar actividad física, caminar, no asistir a programas televisivos que aborden violencia, asistir a películas románticas, valorar el fin de semana como un tiempo para relajar y ocuparse de la familia, y atender a sesiones psicoterápicas; entre otras, fueron estrategias utilizadas por profesionales que atienden a víctimas de violencia para reducir el impacto de las consecuencias sufridas a través del trabajo con fuerte contenido relacional.

En el caso de este trabajo, la investigadora pudo expresar sus dudas en el espacio destinado a tutoría de la investigación en el cual fue asesorada a practicar técnicas de respiración ante situaciones de entrevistas con contenido movilizador. Además, la investigadora mantuvo su rutina de ejercicios físicos, práctica de meditación y actividades de ocio para preservar su bienestar.

\section{Consideraciones Finales}

En este trabajo, se han presentado algunos aspectos que deben plantearse los(as) investigadores(as) antes de llevar a cabo un estudio sobre violencia de género en la pareja. Se han señalado aspectos referidos a la confidencialidad, a la carga emocional que están expuestos los(as) investigadores(as), al estigma que esta temática suscita. Se debe tener en cuenta el momento de vida y la fase del proceso de la violencia que está atravesando las mujeres que consienten participar de estudios sobre violencia. Todo ello afecta a la obtención de una muestra idónea en contexto cualitativo. La consideración de esos factores puede favorecer a las investigaciones una mayor garantía de cualidad.

Las dificultades encontradas en el recurrido metodológico de investigaciones sobre violencia pueden ser un reflejo de los obstáculos (por ejemplo, transporte, horario de las terapias) que impiden que las mujeres busquen apoyo institucional. Ello lleva a entender que hay barreras comunes en el campo de investigación e intervención en violencia de género que merecen ser identificadas y superadas. Además se pudo identificar que la inexistencia de neutralidad y el impacto de la violencia en la subjetividad de la persona que escucha el testimonio de violencia son características que coinciden en la intervención e investigación sobre violencia.

Todo ello lleva a afirmar que se pudo contestar el objetivo del artículo de relatar la experiencia de búsqueda de participantes de una investigación sobre violencia de género en la pareja contra mujeres inmigrantes latinoamericanas y de describir las estrategias utilizadas para obtener el muestreo al poner de manifiesto la dificultad de lograr una muestra según los fundamentos de investigación científica. Otras dificultades fueron la elección por la metodología 
cualitativa debido a los criterios de saturación teórica y el efecto de la violencia en la subjetividad de la investigadora y de la entrevistada.

Tanto la presente investigación sobre violencia cuanto otros trabajos sobre esta temática se construyen teniendo en cuenta los objetivos del estudio, el tiempo de la ayuda financiera facilitada a la investigación y la dificultad de acceso a las participantes. Todo ello señala que el camino realizado para lograr la muestra, recoger, analizar y transcribir los datos "se hace durante el andar", a medida que emergen los obstáculos y se toman decisiones.

\section{Referencias}

Alves, A. J. (1991). O planejamento de pesquisas qualitativas em educação. Cadernos de Pesqui$s a, 77,53-61$.

American Psychological Association. (2010). Publication manual of the American Psychological Association. Washington, DC: Author.

Ayuntamiento de Barcelona. (2011). Informes estadísticos: la población extranjera a Barcelona 2011. Recuperado en http://www.bcn.es/estadistica/catala/dades/inf/pobest/index.htm

Bauer, M., \& Aarts, B. (2004). A construção do corpus: Um princípio para a coleta de dados qualitativos. In A. Bauer \& G. Gaskell (Orgs.), Pesquisa qualitativa com texto, imagem e som: Um manual prático (pp. 39-63). Petrópolis, RJ: Vozes.

Charmaz, K. (2001). Qualitative interviewing and grounded theory analysis. In J. F. Gubrium \& J. A. Holstein (Eds.), Handbook of interview research: Context \& method (pp. 675-694). London: Sage.

Coyle, A. (2007). Introduction to qualitative psychological research. In E. Lyons \& A. Coyle (Eds.), Analysing qualitative data in psychology (pp. 9-29). London: Sage.

Creswell, J. W. (1998). Qualitative inquiry and research design: Choosing among five traditions. London: Sage.

Ellsberg, M., \& Heise, L. (2007). Investigando la violencia contra las mujeres: una guía práctica para la investigación y acción. Managua, Nicaragua: Organización Mundial de la Salud.
Fernández-Martorell, M. (Productora). (2009). ¿No queriais saber por qué las matan? POR NADA [DVD]. Barcelona, España: Canónigo Films.

Francis, J. J., Johnston, M., Robertson, C., Glidewell, L., Entwistle, V., Eccles, M. P., \& Grimshaw, J. M. (2009). What is an adequate sample size? Operationalising data saturation for theorybased interview studies. Psychology \& Health, 25(10), 1229-1245.

Gaskell, G., \& Bauer, M. (2004). Para uma prestação de contas públicas: Além da amostra, da fidedignidade e da validade. In A. Bauer \& G. Gaskell (Orgs.), Pesquisa qualitativa com texto, imagem e som: Um manual prático (pp. 470-490). Petrópolis, RJ: Vozes.

Guest, G., Bunce, A., \& Johnson, L. (2006). How many interviews are enough? An experiment with data saturation and variability. Field Methods, 18(1), 59-82. doi:10.1177/1525822X05279903

Herman, J. (2004). Trauma y recuperación: cómo superar las consecuencias de la violencia. Madrid, España: Espasa Calpe.

Konrad, S. P. (2003). Rompiendo el silencio: manual de entrenamiento para activistas, consejeras y organizadoras latinas. San Francisco, CA: Family Violence Prevention Fund.

Martins, H. H. T. S. (2004). Metodologia qualitativa de pesquisa. Educação e Pesquisa, 30(2), 289300 .

Miles, M. B., \& Huberman, A. M. (1984). Qualitative data analysis: A sourcebook of new methods. London: Sage.

Penso, M. A., Almeida, T. M. C., Brasil, K. C. T., Barros, C. A., \& Brandão, P. L. (2010). O atendimento a vítimas de violência e seus impactos na vida de profissionais da saúde. Temas em Psicologia, 18(1), 137-152.

Reinharz, S., \& Chase, S. E. (2001). Interviewing women. In J. F. Gubrium \& J. A. Holstein (Eds.), Handbook of interview research: Context \& method (pp. 221-238). London: Sage.

Romero, I. (2010). Intervención en violencia de género: consideraciones en torno al tratamiento. Intervención Psicosocial, 19(2), 191-199.

Santos, A. (2009). Mulheres, violência, redes de serviço de referência e suporte psicossocial (Dissertação de mestrado, Universidade Federal de Santa Catarina, Florianópolis, SC, Brasil). 
Schraiber, L. B., Lucas d'Oliveira, A. F. P., \& Couto, M. T. (2009). Violência e saúde: Contribuições teóricas, metodológicas e éticas de estudos da violência contra a mulher. Cadernos de Saúde Pública, 25(2), 205-216.

Secretaria Especial de Políticas para as Mulheres. (2008). II Plano nacional de políticas para as mulheres. Brasília, DF: Autor.

Shetty, S., \& Kaguyutan, J. (2002). Immigrant victims of domestic violence: Cultural challenges and available legal protections. National Online Resource Center on Violence Against Women, 1-8.

Shirwadkar, S. (2004). Canadian domestic violence policy and Indian immigrant women. Violence Against Women, 10(8), 860-879. doi:10.1177/1077801204266310
Taylor, S. J., \& Bogdan, R. (1994). Introducción a los métodos cualitativos de investigación. Barcelona, España: Paidós.

Warren, C. A. B. (2001). Qualitative interviewing. In J. F. Gubrium \& J. A. Holstein (Eds.), Handbook of interview research: Context \& method (pp. 83-101). London. Sage.
Recebido: 24/1/2013

$1^{a}$ revisão: 09/07/2013 Aceite final: 25/07/2013 